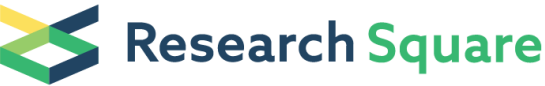 \\ Preprints are preliminary reports that have not undergone peer review. \\ They should not be considered conclusive, used to inform clinical practice, or referenced by the media as validated information.
}

\section{Characterization of Extended-Spectrum $\beta$-Lactamases (ESBLs)-Producing Klebsiella Pneumoniae Strains in Captive Giant Pandas}

\section{Xiaoyan Su}

Chengdu Research Base of Giant Panda Breeding,Sichuan Key Laboratory of Conservation Biology for Endangered Wildlife,Sichuan Academy of Giant Panda, 610081 Chengdu, China

\section{Xia Yan}

Chengdu Research Base of Giant Panda Breeding,Sichuan Key Laboratory of Conservation Biology for Endangered Wildlife,Sichuan Academy of Giant Panda, 610081 Chengdu, China

\section{Yunli Li}

Chengdu Research Base of Giant Panda Breeding,Sichuan Key Laboratory of Conservation Biology for Endangered Wildlife,Sichuan Academy of Giant Panda, 610081 Chengdu, China

\section{Dongsheng Zhang}

Chengdu Research Base of Giant Panda Breeding,Sichuan Key Laboratory of Conservation Biology for Endangered Wildlife,Sichuan Academy of Giant Panda, 610081 Chengdu, China

\section{Lin Li}

Chengdu Research Base of Giant Panda Breeding,Sichuan Key Laboratory of Conservation Biology for Endangered Wildlife,Sichuan Academy of Giant Panda, 610081 Chengdu, China

\section{Yi Geng}

College of Veterinary Medicine, Sichuan Agricultural University, Chengdu 611130, China

\section{Fei Su}

Institute of Animal Husbandry and Veterinary Science, Zhejiang Academy of Agricultural Sciences, Hangzhou, 310021,China

\section{Chanjuan Yue}

Chengdu Research Base of Giant Panda Breeding,Sichuan Key Laboratory of Conservation Biology for Endangered Wildlife,Sichuan Academy of Giant Panda, 610081 Chengdu, China

\section{Rong Hou}

Chengdu Research Base of Giant Panda Breeding,Sichuan Key Laboratory of Conservation Biology for Endangered Wildlife,Sichuan Academy of Giant Panda, 610081 Chengdu, China

\section{Songrui Liu ( $\sim$ srui_liu@163.com )}

Chengdu Research Base of Giant Panda Breeding,Sichuan Key Laboratory of Conservation Biology for Endangered Wildlife,Sichuan Academy of Giant Panda, 610081 Chengdu, China

\section{Research Article}

Keywords: ESBLs-producing K. pneumoniae, antimicrobial resistance, epidemiology, giant panda

Posted Date: February 1st, 2021

DOI: https://doi.org/10.21203/rs.3.rs-144915/v1

License: (c) (1) This work is licensed under a Creative Commons Attribution 4.0 International License. Read Full License 


\section{Abstract}

Background: Extended-spectrum $\beta$-lactamases (ESBLs)-producing strains of Klebsiella pneumoniae remain a critical clinical concern in the world. However, limited information is available concerning ESBLs-producing K. pneumoniae in giant pandas. The objective of this study was to characterize ESBLs-producing K. pneumoniae isolates from the giant panda. A total of 211 K. pneumoniae isolates were collected from 108 giant pandas housed at the Chengdu Research Base of Giant Panda Breeding, China. Samples were screened for the ESBLs-producing phenotype via the double-disk synergy test method.

Result: A total of three $(1.42 \%, \mathrm{n}=211)$ ESBLs-producing K. pneumoniae strains were identified and subjected to MLST (Multi-locus Sequence Typing) analysis for the characterization of ESBLs-encoding genes, transposons, antimicrobial resistance genes, and antimicrobial susceptibility. Among the three ESBLs-producing isolates, different ESBLs-encoding genes, including bla $\mathrm{CTX}_{\mathrm{CT}-\mathrm{M}-1}$, bla $_{\mathrm{SHV}}$, and bla $\mathrm{TEM}_{\mathrm{TEM}}$ were detected. Three isolates were found to carry transposons (i.e., aac6-ib and tnpU) and antimicrobial resistance genes (i.e., aac6-ib, tnpU, aac6-1, qnrA, and qnrB). Furthermore, these three isolates were resistant to at least 15 antibiotics and had different ST isotypes (ST37, ST290, and ST2640).

Conclusion: Effective surveillance and strict infection control strategies should be implemented to prevent outbreaks of ESBLs-producing K. pneumoniae in giant panda.

\section{Introduction}

Klebsiella pneumoniae is an important human pathogen causing numerous infections in hospitals, long-term care facilities, and the associated communities. The bacteria infect the lungs, urinary tract, and surgical sites, causing soft tissue infections and bacteremia (Zhan et al., 2017). The giant panda, Ailuropoda melanoleuca, is one of the world's most recognized and rarest animals and is now only distributed in the mountainous areas of Sichuan, Gansu, and Shaanxi provinces (Wang et al., 2018). The results of the fourth census of giant pandas showed that the population of wild giant pandas reached 1864 ( $\mathrm{Di}$, 2015), and the current population of captive giant pandas reached 600. With the growth of the captive population of giant pandas and the in-depth study of giant panda diseases, infections with K. pneumoniae have emerged. Wang et al. (1998) reported that giant pandas infected with $K$. pneumoniae developed hemorrhagic enteritis. In the same year, a sub-adult giant panda was infected with $K$. pneumoniae and Escherichia coli subsequently died from hemorrhagic sepsis, and the isolated $K$. pneumoniae was found to be pathogenic to mice(Wang \& Wang, 2003). Subsequent cases of genital hematuria, enteritis, and sepsis caused by K. pneumoniae infection in giant pandas were reported (Wang et al, 2006). We conducted an etiological study on a dead giant panda found in a nature reserve in Sichuan and discovered the giant panda died of multiple organ dysfunction syndrome caused by K. pneumoniae and Proteus mirabilis infection(Chen et al., 2020 ). This showed that $K$. pneumoniae infection is a serious threat to the life and health of giant pandas. In addition, studies have shown that $K$. pneumoniae can be transmitted through the air (Bolister et al., 1992), therefore captive animals infected with K. pneumoniae may infect husbandry staff and even visitors to wildlife centers..

The emergence of antibiotic resistance is an increasingly alarming public health threat (Amaya et al., 2011), since it undermines the efficacy of antibiotic treatment and is expected to be the leading cause of global mortality by 2050, possibly exceeding that of cancer (Neill, 2014). $\beta$-lactam antibiotics are among the most frequently prescribed antimicrobials. However, extended-spectrum $\beta$-lactamases (ESBLs) have emerged in numerous hospitals worldwide. These enzymes confer resistance to extended-spectrum cephalosporins and related oxyimino- $\beta$-lactams (ceftazidime, cefotaxime, and aztreonam) but are predominantly sensitive to carbapenems, cephamycins, and $\beta$-lactamase inhibitors such as clavulanic acid (Paterson $\&$ Bonomo, 2005). Antibiotics including $\beta$-endamide are commonly used in disease prevention and control in captive pandas. Statistics for antibiotics use in giant panda indicate that $\beta$-lactam antibiotics accoun for about $50 \%$, while carbapenem antibiotics included imipenem. In the field of human and livestock medicine, experts and researchers have conducted indepth studies on the clinical isolation of ESBLs-producing K. pneumoniae in regards to drug resistance, genetic toxicity, and molecular epidemiology, and these results have played a positive role in the scientific and effective prevention and control of ESBLs-producing K. pneumoniae. However, molecular epidemiology studies of ESBLs-producing K. pneumoniae in giant 
pandas are currently lacking. Therefore, this study aimed to isolate ESBLs-producing K. pneumoniae from captive giant pandas, explore the prevalence and genotype of ESBLs-producing $K$. pneumoniae, provide a scientific basis for the clinical use of antibiotics, and also provide systematic experimental data and a reference basis for preventing and controlling the spread of such bacteria.

\section{Materials And Methods}

\section{Bacterial isolates and screening for ESBLs phenotype}

Two hundred and eleven nonduplicated K. pneumoniae isolates were collected from fresh feces of captive giant pandas from 2018 to 2019 at the Chengdu Research Base of Giant Panda Breeding (Panda Base) in Sichuan, China.. These isolates were identified as K. pneumoniae by Gram staining, 16s rDNA, and bacterial biochemical identification. All of the isolates were tested for ESBLs production by the CLSI-recommended confirmatory double-disc combination test (CLSI, 2019). All of the isolates were screened for ESBLs production using cefotaxime (CTX) and ceftazidime (CAZ) alone and in combination with clavulanic acid according to the double-disk synergy test method (DDST) (CLSI, 2016). Phenotypic presence of ESBLs in the isolates was determined by detecting diameter enhancement of the inhibition zone of the clavulanate disk and corresponding $\beta$-lactam antimicrobial disk. If the enhancement value was $>5 \mathrm{~mm}$, the isolate was presumed to be an ESBLs producer(Yang et al., 2014a; Yang et al., 2014b).

\section{Antimicrobial susceptibility testing}

Antimicrobial susceptibility tests of three ESBLs-producing isolates and seven non-ESBLs-producing isolates were evaluated using the disk diffusion method to Penicillin $1 \mu \mathrm{g}$ (PEN), Piperacillin $100 \mu \mathrm{g}$ (PRL), Ampicillin $10 \mu \mathrm{g}(\mathrm{AMP})$, Oxacillin $1 \mu \mathrm{g}$ (OX), Amoxicillin $20 \mu \mathrm{g}(\mathrm{AML})$, Moxalactam $30 \mu \mathrm{g}$ (MOX), Ceftazidime $30 \mu \mathrm{g}$ (CAZ), Cefixime $5 \mu \mathrm{g}$ (CFM), Cefmatazole $(\mathrm{CMZ})$, Cefepime $30 \mu \mathrm{g}(\mathrm{FEP})$, cephalosporine (CTX), Cephalexin $30 \mu \mathrm{g}(\mathrm{CL})$, Cefazolin $30 \mu \mathrm{g}(\mathrm{KZ})$, Ceftriaxone $30 \mu \mathrm{g}(\mathrm{CRO})$, Cefoxitin $30 \mu \mathrm{g}$ (FOX), Piperacillin/Tazobactam 100/10 $\mu \mathrm{g}$ (TZP), Cefuroxime $30 \mu \mathrm{g}$ (CXM), Cefaclor (CEC), Ampicillin/Sulbactam 10/10 $\mu \mathrm{g}(\mathrm{SAM})$, Cefoperazone $75 \mu \mathrm{g}$ (CFP), Ceftizoxime $30 \mu \mathrm{g}$ (ZOX), Aztreonam $30 \mu \mathrm{g}$ (ATM), Meropenem $10 \mu \mathrm{g}(\mathrm{MEM})$, Imipenem $10 \mu \mathrm{g}(\mathrm{IPM})$, Kanamycin $30 \mu \mathrm{g}(\mathrm{K})$, Streptomycin $10 \mu \mathrm{g}(\mathrm{S})$, Ofloxacin $5 \mu \mathrm{g}(\mathrm{OFX})$, Norfloxacin $10 \mu \mathrm{g}(\mathrm{NOR})$, Ciprofloxacin (CIP); Gatifloxacin $5 \mu \mathrm{g}(\mathrm{GTX})$, Chloramphenicol $30 \mu \mathrm{g}(\mathrm{C})$, Vancomycin $30 \mu \mathrm{g}(\mathrm{VA})$, Azithromycin $15 \mu \mathrm{g}(\mathrm{AZM})$, Doxycycline (TE), Minocycline $30 \mu \mathrm{g}(\mathrm{MH})$, Compound Sulfamethoxazole (SMZ) 23.75/1.25 $\mu \mathrm{g}$, and Trimethoprim $5 \mu \mathrm{g}$ (TMP) (Oxoid, Basingstoke, United Kingdom). The results were interpreted according to the Clinical and Laboratory Standards Institute (CLSI) breakpoints. Escherichia coli ATCC 25922 was used as a control for antimicrobial susceptibility testing.

\section{Multi-locus Sequence Typing (MLST)}

MLST was performed on three ESBLs-producing isolates and 10 non-ESBLs producing isolates by amplifying the seven standard housekeeping loci gapA, infB, mdh, pgi, phoE, rpoB, and tonB as described previously (Diancourt et al. 2005). Sequence types (STs) were assigned using the online database on the Pasteur Institute MLST website (http://bigsdb.pasteur.fr/klebsiella/klebsiella.html).

\section{Molecular investigations of antimicrobial resistance}

All three ESBLs-producing isolates and seven non-ESBLs-producing isolates were screened by PCR for the presence of ESBL

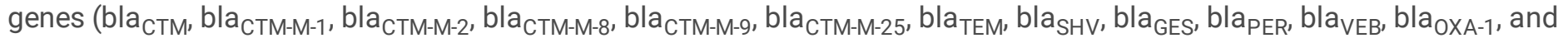

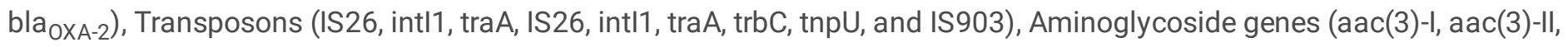
$\operatorname{aac}\left(6^{\prime}\right)-1, \operatorname{aac}\left(2^{\prime \prime}\right)-I, \operatorname{aac}\left(6^{\prime}\right)-I I, \operatorname{tnp} 513$, ant(3")-I, rmtA, rmtB, rmtC, rmtD, armA, aadA5, and npmA), and Quinolones genes (qnrA,

aac(6')-lb, qnrS, and qnrB). The PCR amplicons of bla $\mathrm{CTX}_{\mathrm{C}-\mathrm{M}-1}, \mathrm{bla}_{\mathrm{TEM}}$, and bla $\mathrm{SHE}_{\mathrm{S}}$ from three ESBLs-producing K. pneumoniae isolates were sent to Shanghai Sangong Biotech (Shanghai, China), and sequence analysis was performed using the National Center for Biotechnology Information (NCBI) website with the BLASTn tool. 
Tab. 1 Primers used for detection and sequencing of target genes in Klebsiella pneumoniae isolates. 


\begin{tabular}{|c|c|c|c|c|}
\hline Target & Forward primer $\left(5^{\prime}-3^{\prime}\right)$ & Reverse primer (5'-3') & $\begin{array}{l}\text { Product } \\
\text { size } \\
\text { (bp) }\end{array}$ & $\begin{array}{l}\text { Annealing } \\
\text { temperature } \\
\left({ }^{\circ} \mathrm{C}\right)\end{array}$ \\
\hline \multicolumn{5}{|c|}{$\beta$-Lactamases and ESBLs-encoding genes } \\
\hline CTX-M & ATGTGCAGYACCAGTAARGT & TGGGTRAARTARGTSACCAGA & 593 & 52 \\
\hline $\begin{array}{l}\text { CTX-M- } \\
1\end{array}$ & AAAAATCACTGCGCCAGTTC & AGCTTATTCATCGCCACGTT & 415 & 52 \\
\hline $\begin{array}{l}\text { CTX-M- } \\
2\end{array}$ & CGACGCTACCCCTGCTATT & CCAGCGTCAGATTTTTCAGG & 552 & 52 \\
\hline $\begin{array}{l}\text { CTM- } \\
\text { M-8 }\end{array}$ & TCGCGTTAAGCGGATGATGC & AACCCACGATGTGGGTAGC & 666 & 52 \\
\hline $\begin{array}{l}\text { CTM- } \\
\text { M-9 }\end{array}$ & ATGGTGACAAAGAGAGTGCA & CCCTTCGGCGATGATTCTC & 869 & 52 \\
\hline $\begin{array}{l}\text { CTX-M- } \\
25\end{array}$ & GCACGATGACATTCGGG & AACCCACGATGTGGGTAGC & 327 & 52 \\
\hline TEM & CATTTCCGTGTCGCCCTTATTC & CGTTCATCCATAGTTGCCTGAC & 800 & 52 \\
\hline SHV & AGCCGCTTGAGCAAATTAAAC & ATCCCGCAGATAAATCACCAC & 713 & 52 \\
\hline GES & AGTCGGCTAGACCGGAAAG & TTTGTCCGTGCTCAGGAT & 399 & 52 \\
\hline PER & GCTCCGATAATGAAAGCGT & TTCGGCTTGACTCGGCTGA & 520 & 52 \\
\hline VEB & CATTTCCCGATGCAAAGCGT & CGAAGTTTCTTTGGACTCTG & 648 & 52 \\
\hline \multicolumn{5}{|c|}{ Transposons gene } \\
\hline IS26 & ATGAACCCATTCAAAGGCCGGCAT & TATGCAGCTTTGCTGTTACGACGG & 387 & 55 \\
\hline intl1 & CCGAGGATGCGAACCACTTC & CCGCCACTGCGCCGTTACCA & 373 & 53 \\
\hline $\operatorname{traA}$ & CTGCTGTGGCGGCGTTCTTC & AGTAACCGGCGACCGACATACC & 246 & 53 \\
\hline $\operatorname{trbC}$ & CGGYATWCCGSCSACRCTGCG & GCCACCTGYSBGCAGTCMCC & 255 & 53 \\
\hline $\operatorname{tnpU}$ & GCAAGGAGAAGCGACGAGTGTG & TACATGGCGGTCTCGGCTATCG & 367 & 55 \\
\hline IS903 & GCAATACGCACGCTTTCAGGC & ACTGCACGGTTACGGTCTGCA & 521 & 55 \\
\hline \multicolumn{5}{|c|}{ Aminoglycoside gene } \\
\hline aac(3)-I & АССТАСТСССААСАТСАGСС & ATATAGATCTCACTACGCGC & 169 & 55 \\
\hline$\underset{\text { II }}{\operatorname{aac}(3)-}$ & ACTGTGATGGGATACGCGTC & CTCCGTCAGCGTTTCAGCTA & 237 & 52 \\
\hline aac(6')- & ATGAGTGGCTAAATCGATC & CCCGCTTTCTCGTAGCA & 394 & 55 \\
\hline aac(2")- & GCGAAATCTGCCGCTCTGG & GCTGTTACAACGGACTGGCCG & 319 & 55 \\
\hline$\underset{\text { II }}{\operatorname{aac}\left(6^{\prime}\right)-}$ & TTCATGTCCGCGAGCACCCC & АCTCTTCCGCCATCGCTCTGGG & 178 & 54 \\
\hline $\operatorname{tnp513}$ & CGAGTCAACCTCACACGCTTCC & TGCTCAATGACCTTCGGATCTTCG & 269 & 55 \\
\hline ant(3")- & TGATTTGCTGGTTACGGTGAC & CGCTATGTTCTCTTGCTTTTG & 284 & 55 \\
\hline rmtA & CTAGCGTCCATCCTTTCCTC & $\begin{array}{l}\text { TTTGCTTCCATGCCCTTGCC } \\
\text { Page 5/11 }\end{array}$ & 634 & 55 \\
\hline
\end{tabular}




\begin{tabular}{|lllll|}
\hline rmtB & CCCAAACAGACCGTAGAGGC & CTCAAACTCGGCGGGCAAGC & 565 & 55 \\
\hline rmtC & CGAAGAAGTAACAGCCAAAG & ATCCCAACATCTCTCCCACT & 800 & 55 \\
\hline rmtD & CGGCACGCGATTGGGAAGC & CGGAAACGATGCGACGAT & 750 & 55 \\
\hline armA & AGGTTGTTTCCATTTCTGAG & TCTCTTCCATTCCCTTCTCC & 590 & 55 \\
\hline aadA5 & ATGGGTGAATTYTTYCCTGCACAA & TCAACGCAAGATTCTCTCATTCGT & 789 & 55 \\
\hline npmA & CGGGATCCAAGCACTTTCATACTGACG & CGGAATTCCAATTTTGTTCTTATTAGC & 334 & 54 \\
\hline Quinolones gene & & 578 & 53 \\
\hline qnrA & AGAGGATTTCTCACGCCAGG & TGCCAGGCACAGATCTTGAC & 57 & 53 \\
\hline $\begin{array}{l}\text { aac(6')- } \\
\text { lb }\end{array}$ & TGACCTTGCGATGCTCTATG & TTAGGCATCACTGCGTGTT & 497 & 53 \\
\hline qnrS & GCAAGTTCATTGAACAGGGT & TCTAAACCGTCGAGTTCGGCG & 191 & 55 \\
\hline qnrB & GGMATHGAAATTCGCCACTG & TTTGCYGYYCGCCAGTCGAA & 263 \\
\hline
\end{tabular}

\section{Results}

\section{Prevalence of ESBLs-producing isolates and ESBLs-encoding genes}

The prevalence of ESBLs-producing isolates was 1.42\% $(n=211)$ in the giant pandas listed in the Panda Base (Tab.2).

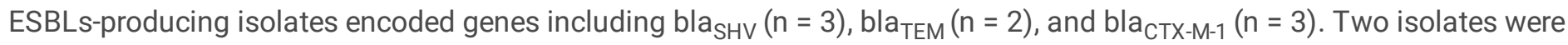
detected co-carrying bla $\mathrm{SHV}_{\text {, }}$ bla $\mathrm{TEM}_{\mathrm{TE}}$, bla $_{\mathrm{CTX}-\mathrm{M}-1}$, and one co-carrying bla $\mathrm{SHV}_{\mathrm{SHV}}$ and bla $\mathrm{C}_{\mathrm{CTX}-\mathrm{M}-1}$ (Tab.3).

Tab. 2 Screening for ESBLs phenotype in isolates

\begin{tabular}{|lllll|}
\hline Isolates & \multicolumn{2}{l}{ inhibition zone $/ \mathrm{mm}$} & \multicolumn{2}{c|}{ Result } \\
\cline { 2 - 4 } & cefotaxime & cefotaxime / Clavulanic acid & value & \\
\cline { 1 - 4 } X1 & 10.43 & 18.16 & 7.73 & positive \\
RJ & 0.00 & 17.74 & 17.74 & positive \\
X2 & 15.01 & 24.97 & 9.96 & positive \\
\hline
\end{tabular}

Tab. 3 Prevalence of ESBLs-encoding genes in ESBLs-producing isolates from giant pandas $(n=211)$

\begin{tabular}{|llll|}
\hline Genes & Isolates & Number of isolates & Percentage of isolates \\
\hline bla $_{\text {TEM }}$ & $\mathrm{X} 1, \mathrm{X} 2$ & 2 & $0.09 \%$ \\
\hline bla $_{\mathrm{SHV}}$ & $\mathrm{X} 1, \mathrm{X} 2, \mathrm{RJ}$ & 3 & $1.42 \%$ \\
bla $_{\mathrm{CTX}-\mathrm{M}-1}$ & $\mathrm{X} 1, \mathrm{X} 2, \mathrm{RJ}$ & 3 & $1.42 \%$ \\
\hline
\end{tabular}

\section{Antimicrobial resistance profiles}

The three ESBLs-producing isolates were all resistant to penicillin (100\%), piperacillin (100\%), cephalosporine (100\%), cephalexin, cefazolin, ceftriaxone, cefuroxime, cefaclor, cefoperazone, kanamycin, streptomycin, vancomycin, doxycycline, 
and compound sulfamethoxazole(Fig. 1). Isolate X1 was resistant to 18 antibiotics. Isolate X2 was resistant to 15 antibiotics while isolate RJ was resistant to 27 antibiotics, however, seven non-ESBLs-producing isolates were resistant to vancomycin (100\%) and imipenem (75\%).

\section{Molecular characteristics of ESBLs-producing isolates}

ESBLs-producing Isolates X2 and X1 were detected co-carrying three ESBLs encoding genes (CTX-M1, TEM, and SHV), while RJ co-carried two ESBLs-encoding genes (CTX-M1 and SHV). Among the three ESBL isolates, three STs were identified, ST2640, ST290, and ST37. Genetic phylogenetic tree analysis showed that ESBLs-positive isolates X2 and X1 belonged to the same cluster (Fig. 2).

\section{Transposons and antimicrobial resistance genes distribution}

All three ESBLs-producing isolates and seven non-ESBLs-producing isolates tested were carrying at least one of the 22 investigated transposons and antimicrobial resistance genes. Three ESBLs-producing isolates were carrying five antimicrobial resistance genes and 6-8 transposons. Seven non-ESBLs-producing isolates were carrying 1-3 antimicrobial resistance genes and three transposons. All of the isolates were carrying aac6-ib and tnpU. Compared with the seven non-ESBLs-producing isolates, the three ESBLs-producing isolates carried aadA5, rmtD, IS903, and intl1 (Fig. 2).

\section{Discussion}

ESBL-producing K. pneumoniae have been frequently encountered worldwide, especially in hospitals. However, ESBLsproducing $K$. pneumoniae are rarely found in wild animals such as the giant panda. Therefore, this study intended to analyze the prevalence, genotype, and antimicrobial susceptibility profiles of ESBL-producing K. pneumoniae collected from captive giant pandas to provide a scientific basis for the clinical use of antibiotics, and also to provide a systematic data and reference base for preventing and controlling the spread of such bacteria. In this study, the prevalence of ESBLs-producing $K$. pneumoniae among $K$. pneumoniae isolates $(n=211)$ from giant pandas was $1.42 \%$. The prevalence of ESBLs-producing $K$. pneumoniae in giant pandas was lower than in humans. The most common ESBL-producing Enterobacteriaceae detected in a Children's hospital in Japan was E. coli (79.8\%), followed by K. pneumoniae (9.1\%) (Yamanaka et al. 2020). In Iran, the prevalence of ESBL-producing K.pneumoniae was 43.5\% (95\% Cl 39.3-47.9\%) among clinical K. pneumoniae isolates (Beigverdi et al., 2019a). In a study from Sweden in 2018, Hessman et al. (2018) reported that only $2 \%$ of K. pneumoniae isolates produced ESBLs. In Spain, the prevalence of ESBL-producing K. pneumoniae in 2017 was 7.2\% (Cubero et al., 2018). In a similar study conducted in Canada from 2010-2012, Karlowsky et al. (2013) reported that the prevalence of ESBL producers was 3.6\% among K. pneumoniae isolates. Studies in Saudi Arabia, United Arab Emirates, and Tunisia between 2009 to 2018 revealed a prevalence of ESBL-producing K. pneumoniae between 38\% and 55\% in different community settings and hospitals (Beigverdi et al., 2019b). A possible explanation for the prevalence of ESBLs-producing K. pneumoniae in giant pandas was lower is that the use of antibiotics is less frequent in giant pandas than in humans.

In this study, three ESBL-producing K. pneumoniae isolates were detected co-carrying 2 3 ESBLs-encoding genes, including

bla $_{\mathrm{TEM}}$, bla $\mathrm{S}_{\mathrm{SHV}}$, and bla $\mathrm{C}_{\mathrm{CTX}-\mathrm{M}-1}$. This result was similar to that described in previous studies where TEM- and CTX-M-types were the main families of ESBLs (Paterson \& Bonomo 2005; Bush \& Jacoby 2010). Furthermore, reports have indicated that CTX-Ms have been rapidly disseminated among populations of Gram-negative bacteria in clinical settings in recent years (Salverda et al., 2010; Seiffert et al., 2013).

Three ESBL-producing K. pneumoniae isolates showed high resistance to many antimicrobial agents (more than 15 antibiotics). In our study, Meropenem was effective against three ESBLs-producing K. pneumoniae isolates, and IPM was effective against about $10 \%$ of the strains $(n=10)$. Three ESBLs-producing $K$. pneumoniae isolates were carrying five antimicrobial resistance genes, such as aac6-ib, tnpU, aac6-1, qnrA, and qnrB, which are associated with aminoglycoside and quinolones resistance. In China, ESBLs-positive K. pneumoniae strains were $>70 \%$ susceptible only to IPM, ertapenem, or amikacin (Zhang et al., 2017). Similar similar studies have reported that although the majority of ESBLs-producing strains

Page $7 / 11$ 
could tolerate high concentrations of most cephalosporins and fluoroquinolones, none exhibited resistance to carbapenems (i.e., imipenem and meropenem) (Qiao et al., 2017). Interestingly, some of these non-ESBLs-producing K. pneumoniae strains were detected carrying aminoglycoside and quinolones-encoding genes that are associated with aminoglycoside and quinolones resistance, but none of them exhibited resistance to those antimicrobial agents. The reason may be partly due to the expression levels of aminoglycoside and quinolones-encoding genes being quite low in most cases (Poirel \& Nordmann, 2006).

Some studies have reported that about $50 \%$ of the antibiotic prescriptions for the treatment of K. pneumonia infection are inappropriate and may cause the emergence of antimicrobial resistance (AMR) (Lee et al., 2013). We recommend that veterinary professionals pay close attention to the choice of antimicrobial agents to prevent widespread AMR emergence in captive giant pandas.

Transposons are transposable genetic elements that harbor resistance genes. Transposons were found to be involved in the spread and development of drug resistance in Gram-negative bacteria. The greater the number and types of transposons, the greater the potential for drug resistance transmission (Wellington et al., 2013; Kelly et al., 2009; Domingues et al., 2012). ESBLs-producing K. pneumoniae strains in this study carried eight different types of transposons, while non-ESBLsproducing K. pneumoniae strains carried significantly fewer transposons (2-3). The results indicated that ESBLs-producing K. pneumoniae strains had a stronger ability to spread drug resistance.

Our study assessed the genotypes of three ESBL-producing $K$. pneumoniae using MLST, and the analysis revealed that three ESBLs-producing isolates had different ST isotypes (ST37, ST290, and ST2640). ST37 and ST290 had a closer phylogenetic relationship.

\section{Conclusions}

In summary, three $(1.42 \%)$ out of the $211 \mathrm{~K}$. pneumoniae isolates recovered from giant pandas were confirmed as ESBLs positive. Among the three ESBLs-producing isolates, different ESBLs-encoding genes, including bla $\mathrm{C}_{\mathrm{CTX}-\mathrm{M}-1}, \mathrm{bla}_{\mathrm{SHV}}$, and bla $\mathrm{T}_{\mathrm{TEM}}$, were detected. Furthermore, three isolates were found to carry transposons (i.e., aac6-ib and tnpU) and antimicrobial resistance genes, (i.e., aac6-ib, tnpU, aac6-1, qnrA, and qnrB). These three isolates were resistant to at least 15 antibiotics and had different ST isotypes (ST37, ST290, and ST2640). We recommend that clinical veterinarians pay more attention to the choice of antimicrobial agents to prevent widespread ESBLs-producing $K$. pneumoniae emergence in the giant panda.

\section{Declarations}

The research in the manuscript has been conducted under the guidance of the Institutional Animal Care and Use Committee of the Chengdu Research Base of Giant Panda Breeding.

\section{Consent for publication}

The manuscript is approved by all authors for publication. The work described was original research that has not been published previously, and not under consideration for publication elsewhere, in whole or in part.

\section{Ethics approval and consent to participate}

The use of materials and all experimental procedures involving animals were approved by Institutional Animal Care and Use Committee of the Chengdu Research Base of Giant Panda Breeding protocol \#2018017.

\section{Availability of data and materials}

All data generated or analysed during this study are included in this published article and its supplementary information files. 


\section{Competing interests}

The authors declare that they have no competing interests.

\section{Author Contributions:}

All authors read and approved the final manuscript. Xiaoyan Su: Conceptualization, Formal analysis, funding acquisition, methodology, investigation, validation, project administration, resources, writing-original draft, writing-review and editing, project administration; Xia Yan: methodology and Formal analysis; Yunli Li: Investigation; Dongsheng Zhang: Resources; Lin Li: software; Fei Su, Yi Geng and Chanjuan Yue: Methodology; Rong Hou: funding acquisition; Songrui Liu: Writing-review \& editing

Conflicts of Interest: Not applicable.

Funding: This research was funded by Sichuan Science and Technology Program (2018JY0363) and Chengdu Giant Panda Breeding Research Foundation (CPF2017-18).

Acknowledgements: The authors thank all the staff involved in the animal feeding and thank James Edward Ayala for the assistance in English editing.

\section{References}

1. Amaya E., Reyes D., Vilchez S., Paniagua M., Möllby R., Nord C.E. \& Weintraub A. Antibiotic resistance patterns of intestinal Escherichia coli isolates from Nicaraguan children. J Med Microbio/ 2011, 60, 216-222.

2. Beigverdi R., Jabalameli L., Jabalameli F. \& Emaneini M. Prevalence of extended-spectrum $\beta$-lactamase-producing Klebsiella pneumoniae: First systematic review and meta-analysis from Iran. J Glob Antimicrob Resist 2019, 18, $12-21$.

3. Bolister N.J., Johnson H.E. \& Wathes C.M.. The ability of airborne Klebsiella pneumoniae to colonize mouse lungs. Epidemiol Infect 1992, 109, 121-131.

4. Bush K. \& Jacoby G.A. Updated functional classification of beta-lactamases. Antimicrob Agents Chemother 2010, 54, 969-76.

5. Chen Y. Y., Liu S.R., Su X.Y., Geng Y., Huang W.J., Chen X., Bai M.H. \& Cheng Z.X. A case of Klebsiella pneumoniae and Proteus mirabilis infection in giant panda (Ailuropoda melanoleuca).Chinese Journal Of Wildlife 2020,41(04):1013-19.

6. Cubero M., Grau I., Tubau F., Pallarés R., Domínguez M.Á., Liñares J. \& Ardanuy C. Molecular Epidemiology of Klebsiella pneumoniae Strains Causing Bloodstream Infections in Adults. Microb Drug Resist 2018,24, 949-957.

7. Di G.H. 1864 Wild Pandas Protection in China Makes New Achievements. Lü se Zhongguo 2015, 10-12.

8. Diancourt L., Passet V., Verhoef J., Grimont P.A. \& Brisse S. Multilocus sequence typing of Klebsiella pneumoniae nosocomial isolates. J Clin Microbio/ 2005, 43, 4178-82.

9. Domingues S., Harms K., Fricke W.F., Johnsen P.J., Da S.G. \& Nielsen K.M. Natural transformation facilitates transfer of transposons, integrons and gene cassettes between bacterial species. Plos Pathog 2012, 8, e1002837.

10. Hessman J., Atterby C., Olsen B. \& Järhult J.D. High Prevalence and Temporal Variation of Extended Spectrum $\beta$ Lactamase-Producing Bacteria in Urban Swedish Mallards. Microb Drug Resist 2018, 24, 822-829.

11. Karlowsky J.A., Adam H.J., Baxter M.R., Lagacé-Wiens P.R., Walkty A.J., Hoban D.J. \& Zhanel G.G. In vitro activity of ceftaroline-avibactam against gram-negative and gram-positive pathogens isolated from patients in Canadian hospitals from 2010 to 2012: results from the CANWARD surveillance study. Antimicrob Agents Chemother 2013, 57, 5600-5611.

12. Kelly B.G., Vespermann A. \& Bolton D.J. The role of horizontal gene transfer in the evolution of selected foodborne bacterial pathogens. Food Chem Toxicol 2009, 47, 951-968.

13. Lee C.R., Cho I.H., Jeong B.C. \& Lee S.H. Strategies to minimize antibiotic resistance. Int J Environ Res Public Health 2013, 10, 4274-4305. 
14. Neill O.. Antimicrobial Resistance: Tackling a crisis for the health and wealth of nations. 2014.

15. Paterson D.L. \& Bonomo R.A.. Extended-spectrum beta-lactamases: a clinical update. Clin Microbiol Rev 2005, 18, 657686.

16. Poirel L. \& Nordmann P. Carbapenem resistance in Acinetobacter baumannii: mechanisms and epidemiology. Clin Microbiol Infect 2006, 12, 826-36.

17. Qiao J, Zhang Q, Alali WQ, Wang J, Meng L, Xiao Y, Yang H, Chen S, Cui S \& Yang B. Characterization of extendedspectrum $\beta$-lactamases (ESBLs)-producing Salmonella in retail raw chicken carcasses. Int J Food Microbio/ 2017, 2, 248:72-81.

18. Salverda M.L., De Visser J.A. \& Barlow M. Natural evolution of TEM-1 $\beta$-lactamase: experimental reconstruction and clinical relevance. Fems Microbiol Rev 2010, 34, 1015-36.

19. Seiffert S.N., Hilty M., Perreten V. \& Endimiani A. Extended-spectrum cephalosporin-resistant Gram-negative organisms in livestock: an emerging problem for human health? Drug Resist Updat 2013, 16, 22-45.

20. Wang C.D., Lan J.C. \& Luo L. Giant panda infectious genitourinary hematuria pathogen-Klebsiella pneumoniae. Sichuan Journal of Zoology 2006, 25, 83.

21. Wang Q, Jiang H, Tateko Nakao \& Koji Imazu. A case report of giant panda Klebsiella pneumoniae hemorrhagic enteritis. Sichuan Journal of Zoology 1998,17(1):29.

22. Wang T., Xie Y., Zheng Y., Wang C., Li D., Koehler A.V. \& Gasser R.B. Parasites of the Giant Panda: A Risk Factor in the Conservation of a Species. Adv Parasitol 2018, 99, 1-33.

23. Wang X.Y. \& Wang Y. Second Giant Panda Germs Sapraemia Diagnosis. Sichuan Animal \& Veterinary Sciences $2003,20$.

24. Wellington E.M., Boxall A.B., Cross P., Feil E.J., Gaze W.H., Hawkey P.M., Johnson-Rollings A.S., Jones D.L., Lee N.M., Otten W., Thomas C.M. \& Williams A.P. The role of the natural environment in the emergence of antibiotic resistance in gram-negative bacteria. Lancet Infect Dis 2013, 13, 155-65.

25. Yamanaka T., Funakoshi H., Kinoshita K., Iwashita C. \& Horikoshi Y. CTX-M group gene distribution of extended spectrum beta-lactamase-producing Enterobacteriaceae at a Japanese Children's hospital. J Infect Chemother 2020, 26, 1005-7.

26. Yang B., Cui Y., Shi C., Wang J., Xia X., Xi M., Wang X., Meng J., Alali W.Q., Walls I. \& Doyle M.P.. Counts, serotypes, and antimicrobial resistance of Salmonella isolates on retail raw poultry in the People's Republic of China. J Food Prot 2014a, 77, 894-902.

27. Yang B., Wang Q., Cui S., Wang Y., Shi C., Xia X., Xi M., Wang X., Shi X., Wang D., Zhang Z. \& Meng J.. Characterization of extended-spectrum beta-lactamases-producing Salmonella strains isolated from retail foods in Shaanxi and Henan Province, China. Food Microbio/ 2014b, 42, 14-18.

28. Zhan L., Wang S., Guo Y., Jin Y., Duan J., Hao Z., Lv J., Qi X., Hu L., Chen L., Kreiswirth B.N., Zhang R., Pan J., Wang L. \& Yu F. Outbreak by Hypermucoviscous Klebsiella pneumoniae ST11 Isolates with Carbapenem Resistance in a Tertiary Hospital in China. Front Cell Infect Microbio/ 2017, 7, 182.

29. Zhang H., Yang Q., Liao K., Ni Y., Yu Y., Hu B., Sun Z., Huang W., Wang Y., Wu A., Feng X., Luo Y., Chu Y., Chen S., Cao B., Su J., Duan Q., Zhang S., Shao H., Kong H., Gui B., Hu Z., Badal R. \& Xu Y. Update of incidence and antimicrobial susceptibility trends of Escherichia coli and Klebsiella pneumoniae isolates from Chinese intra-abdominal infection patients. BMC Infect Dis 2017. 17(1):776.

\section{Figures}




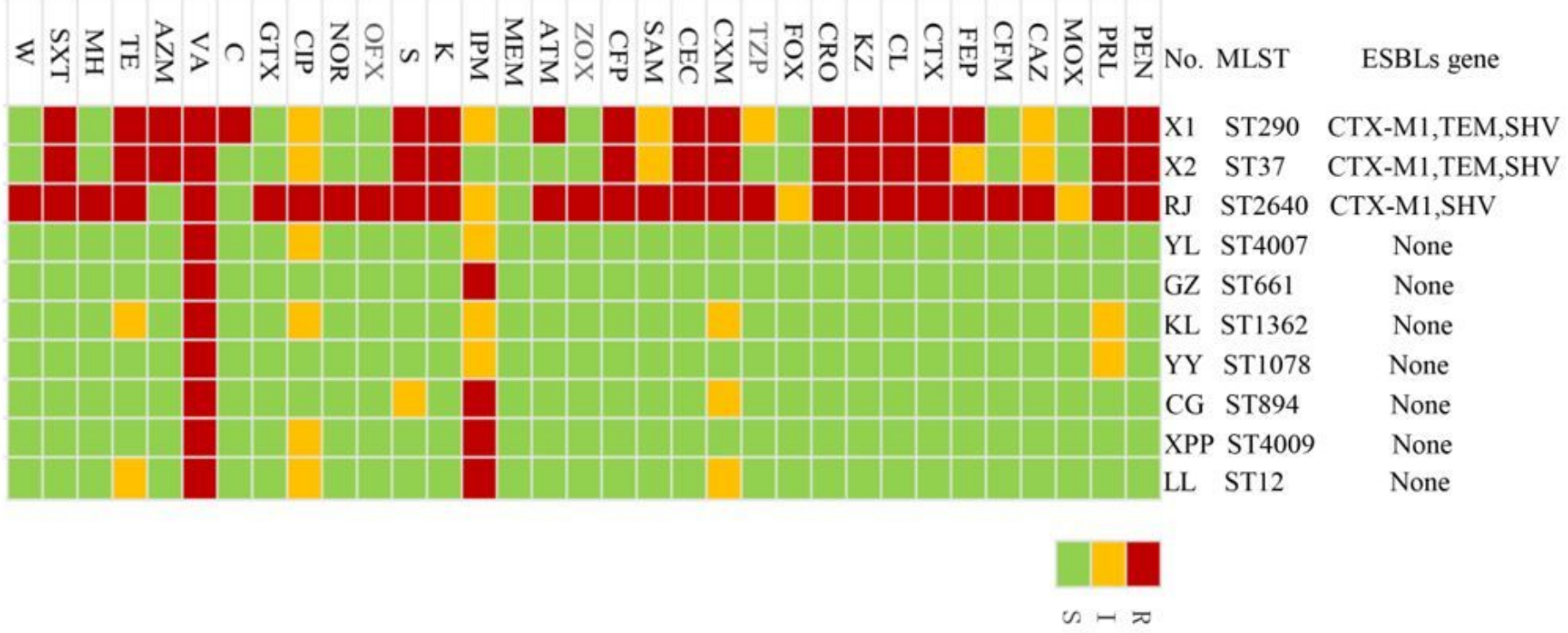

\section{Figure 1}

Antimicrobial susceptibility profiles of Kp isolates. $\mathrm{X} 1, \mathrm{X} 2$, and $\mathrm{RJ}$ are ESBLs- positive Klebsiella pneumoniae isolates. $\mathrm{YL}, \mathrm{GZ}$, $\mathrm{KL}, \mathrm{YY}, \mathrm{CG}, \mathrm{XPP}$, and LL are non- ESBLs positive Klebsiella pneumoniae isolates as controls. PEN, Penicillin; PRL, Piperacillin; AMP, Ampicillin; OX, Oxacillin; AML, Amoxicillin; MOX, Oxycephalosporin; CAZ, Ceftazidime; CFM, Cefixime; CMZ, cefmatazole; FEP, Cefepime; CTX, cephalosporine; CL, Cephalexin; KZ, Cefazolin; CRO, Ceftriaxone; FOX, Cefoxitin; TZP, Piperacillin/Tazobactam; CXM, Cefuroxime; CEC, Cefaclor; SAM, Ampicillin/Sulbactam; CFP, Cefoperazone; ZOX, Ceftizoxime; ATM, Aztreonam; MEM, Meropenem; IPM, Imipenem; K, Kanamycin; S, Streptomycin; OFX, Ofloxacin; NOR, Norfloxacin; CIP, Ciprofloxacin; GTX, Gatifloxacin; C, Chloramphenicol; VA, Vancomycin; AZM, Azithromycin; TE, Doxycycline; MH, Minocycline; SXT, Compound Sulfamethoxazole; TMP, trimethoprim.

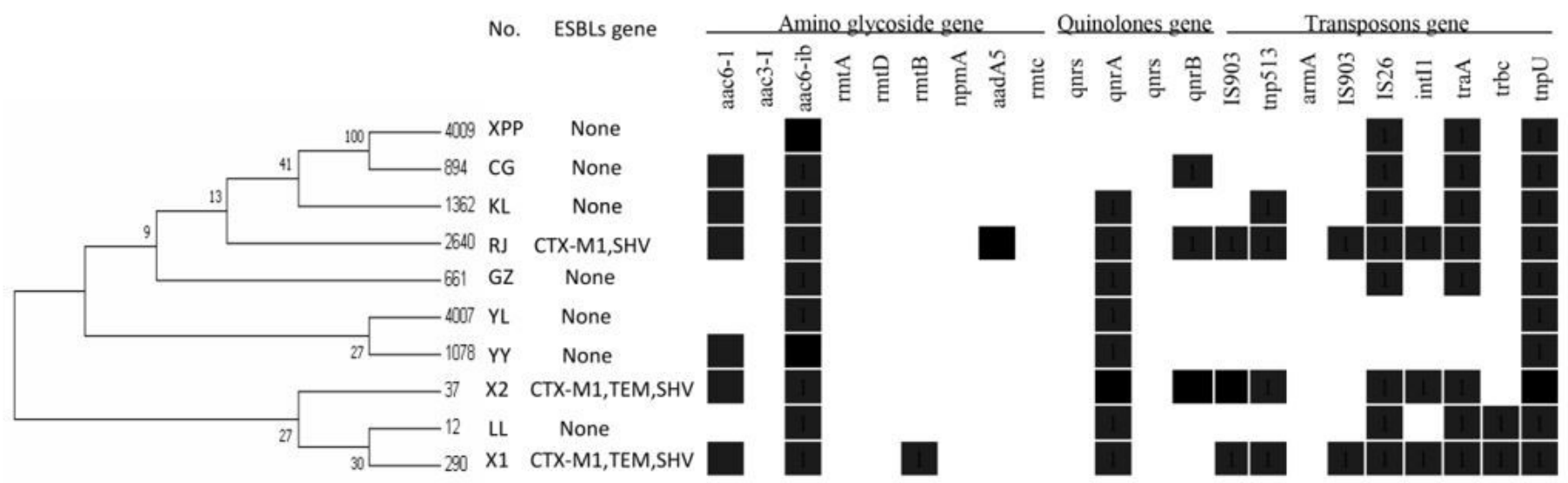

Figure 2

Dendrogram and genetic features of 10 isolates from giant pandas recovered in 2018 and 2019 with the respective ESBLs genes, transposons, and antimicrobial resistance genes. X1, X2, and RJ are ESBLs- positive Klebsiella pneumoniae isolates. YL, GZ, KL, YY, CG, XPP, and LL are non- ESBLs positive Klebsiella pneumoniae isolates as controls. 